Preliminary version in Proceedings of The 9th RoboCup International Symposium, Osaka, Japan, paper \#97, July 2005.

\title{
Playing Soccer with RoboSapien
}

\author{
Sven Behnke, Jürgen Müller, and Michael Schreiber \\ Albert-Ludwigs-University of Freiburg, Computer Science Institute \\ Georges-Köhler-Allee 52, 79110 Freiburg, Germany \\ \{ behnke|jmuller | schreibe \} @ informatik.uni-freiburg.de
}

\begin{abstract}
Due to limited availability of humanoid robots and the high costs involved, multi-agent experiments with humanoid robots have been at least difficult so far. With the introduction of RoboSapien, a low-cost humanoid robot developed for the toy market, this situation has changed. This paper describes how we augmented multiple RoboSapiens to obtain a team of soccer playing humanoid robots. We added a Pocket PC and a color camera to the robot base to make it autonomous.

For a team of these augmented RoboSapiens, we implemented computer vision and self localization. We designed basic soccer skills, such as approaching the ball, dribbling the ball towards the goal, and defending the goal. We set up a soccer field and played test games in our lab to evaluate the system.

The paper reports experiences made during these soccer matches as well as results on a scoring task. We also tested this system at RoboCup German Open 2005, where we played soccer matches against the Brainstormers Osnabrück, who also used augmented RoboSapiens.
\end{abstract}

\section{Introduction}

To work towards the long-term goal of winning against the FIFA world champion, the RoboCup Federation added in 2002 a league for humanoid robots to their annual soccer championships. The RoboCup Humanoid League competition rules [14] require the participating robots to have a human-like body plan. They must consist of a trunk, two legs, two arms, and a head. The only allowed mode of locomotion is bipedal walking. The robots must be fully autonomous. No external power, computing power, or remote control is allowed.

Because the humanoid robots have not been ready for playing soccer games so far, the robots had to demonstrate their capabilities by solving a number of subtasks. In the Humanoid Walk they had to walk towards a pole, to turn around it, and to come back to the start. Scoring was based on walking speed and stability. In the Penalty Kick competition two robots faced each other. While one robot tried to score a goal, the other defended. In the Freestyle competition, the robots had five minutes to show a performance to a jury. Each year, there is also a new technical challenge. In 2004, it consisted of an obstacle walk, a passing task, and balancing across a sloped ramp.

The teams which participated in the Humanoid League chose very different robot platforms. Most teams constructed their own robots (e.g. Robo-Erectus [30]). 
A few teams used expensive humanoid robots developed by the Japanese industry, e.g. Hoap-2 [16] or Honda Asimo [8]. Some teams purchased servo-driven commercial robots or robot kits, e.g. from iXs [10] or Vstone [26].

The performance of the robots in the Humanoid League improved over the three competitions. In 2004, Team Osaka won the competition with the robot VisiON [21]. This robot used an omnidirectional camera as head. As a goalie, it could defend against a shot by jumping to the ground. Afterwards, VisiON got up without help. Another highlight of the 2004 competition was the passing demonstration between two Hoap-2 robots of team Senchans A [16].

Despite these impressive achievements, the overall performance of the RoboCup humanoids is still far from perfect. Basic soccer skills, such as robust dynamic walking and kicking without loosing balance are not possessed by all robots. Moreover, due to the high price involved, it exceeds the resources of most research groups to buy or construct more than one robot. To play soccer, however, a group needs a number of players in order to field a team.

Fortunately, RoboSapien, a low cost commercial humanoid robot, hit the market in 2004. In its original version, it is controlled by a human operator. We found a way to make it autonomous by augmenting it with a Pocket PC and a camera. Due to the low cost of this solution, it is not hard to obtain multiple augmented RoboSapiens. Since basic problems, such as dynamic walking, are solved when using this robot base, one can focus on higher-level issues, such as visual perception, self localization, behavior control, and communication.

The paper is organized as follows. The next section reviews some of the related work. Section 3 presents the original RoboSapien. In Section 4, we describe how we augmented it with a Pocket PC and a camera. Section 5 covers visual perception and self localization on the soccer field. Behavior control for making it play soccer is detailed in Section 6. In Section 7, we describe some infrastructure components needed to support a team of soccer playing robots. Section 8 reports experiences made during test games and presents experimental results on a scoring test. The paper concludes with a discussion of the feasibility of using RoboSapien for soccer competitions.

\section{Related Work}

Humanoid robots are not only used to play soccer. The human-like body has advantages when the robots are used in environments designed for humans. It facilitates multimodal communication with humans and imitation learning. Consequently, a number of research groups, especially in Japan, are constructing humanoid robots. A list of projects is maintained by Willis [28].

Among the most advanced humanoid robots developed so far, is the $58 \mathrm{~cm}$ tall Sony Qrio [19]. It contains three CPUs and has 38 degrees of freedom (DOF). Qrio is able to walk and dance. Research on map building and navigation, as well as on human-robot interaction is carried out inside Sony. Currently, it is unclear if and when this robot will be available to a larger research community, but the costs of Qrio have been compared to the price of a luxury car. 
Unlike Qrio, Hoap-2 (25 DOF, 50cm tall), developed by Fujitsu [7], has been sold to some labs for about USD 50,000. A taller humanoid, Asimo, has been developed by Honda [8]. Its most recent research version has 34 DOFs and a height of $130 \mathrm{~cm}$. Approximately the same size of Asimo has a trumpet playing humanoid robot which has been announced recently by Toyota [24].

While the humanoid robots developed by large companies are impressive, they are not available to researchers outside the industry labs or are too expensive for academic research. Some universities built their own robots, but due to limited resources, usually only one prototype has been constructed. Hence, multirobot experiments with humanoid robots are currently not feasible in academic environments and are likely to be at least difficult in the near future.

Faced with similar problems, researchers working with wheeled robots came up with creative low-cost solutions. One example of a low-cost robot kit is the Lego Mindstorms system. It has been used e.g. for robotic soccer [13], education [29], and communication with people [11]. Other low-cost robotic platforms include the Tetrixx kit [5], the Trikebot [9], and the VolksBot [1].

To avoid the development of custom processing boards, some researchers used off-the-shelf PDAs to control their robots $[27,15]$. One of the best know PDA projects is the Palm Pilot Robot Kit $[4,17]$, developed at CMU. A PDA has also been used to control the Robota and DB humanoid robots [3].

While RoboSapien is certainly the most frequently sold humanoid robot today, it is not the only option for researchers. Kondo developed the servo-driven KHR-1 robot kit [12]. We augmented it with a Pocket PC and a camera as well and use it for gait optimization and to play soccer. Compared to RoboSapien, it is more expensive ( $\approx$ EUR 1,000), less robust, and less stable. On the other hand, it has more degrees of freedom (17) than RoboSapien and can move in a more flexible way.

Similar servo-driven robots are offered from Vstone (Robovie-M/MS [26]), Tribotix (Cycloid [25]), and Speecys [20]. Vstone also offers the VisiON robot (Robovie-V) for a price of approximately EUR 7,200. A question for further research would be to find out if the higher number of DOFs of these robots translates to better performance in soccer games. One possible danger could be that walking stability is compromised in these more complex designs.

\section{Original RoboSapien}

RoboSapien, shown in Fig. 1, is a low-cost humanoid robot, which has been designed by Mark W. Tilden [23] and is marketed with great success by WowWee for the toy market. It measures approximately $34 \mathrm{~cm}$ in height and its weight is about $2.1 \mathrm{~kg}$, including four mono (D) type batteries. These batteries are located in its feet. The low center of mass makes RoboSapien very stable.

The robot is driven by seven small DC motors. One motor per leg moves two joints in the hip and the knee in the sagittal plane, keeping the foot orthogonal to the trunk. A trunk motor tilts the upper body laterally. One motor in each shoulder raises and lowers the arm and one motor in each elbow twists the lower 

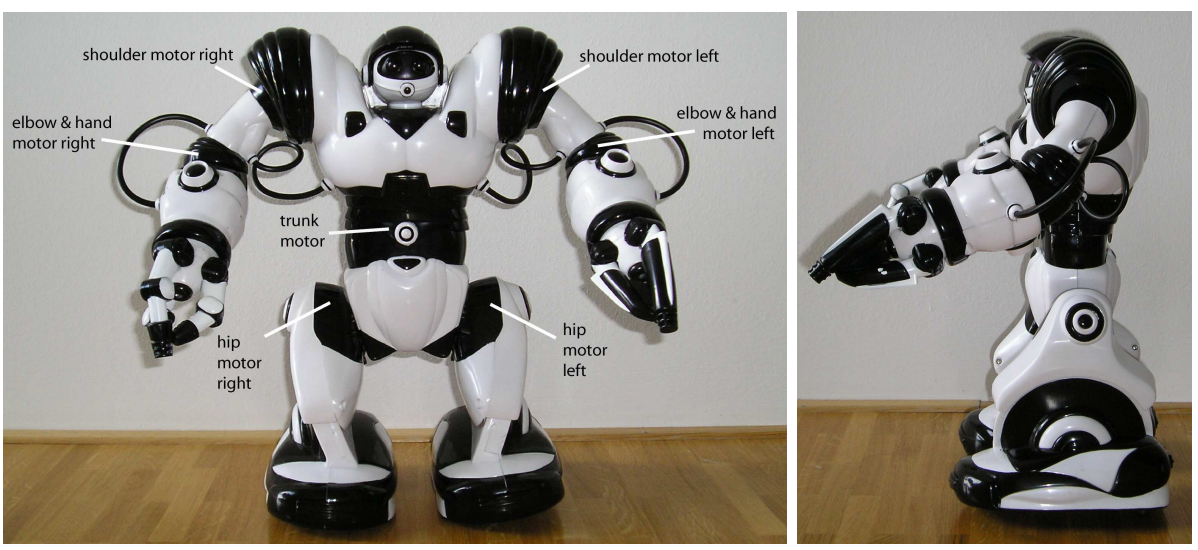

Fig. 1. Robo Sapien. Left: frontal view, seven motors move the robot. Right: side view.
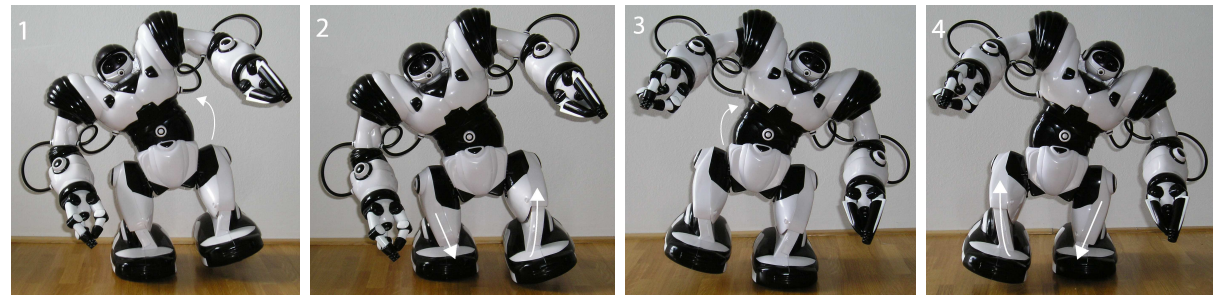

Fig. 2. Dynamic walking gait of RoboSapien. (1) The trunk motor tilts the upper body to the right. The center of mass shifts over the right foot. The left foot lifts from the ground. (2) The leg motors move into opposite directions, resulting in a forward motion of the robot. As the upper body swings back, the left foot regains contact with the ground. $(3,4)$ Symmetrical to $(1,2)$.

arm and opens its grippers. RoboSapien has two gripper hands consisting of three fingers each.

Unlike more complex bipedal robots, RoboSapien uses only three motors for locomotion. This is possible because its gait patterns utilize the dynamics of the robot. For dynamic walking, RoboSapien swings its upper body laterally to achieve a periodic displacement of the center of mass projection from one foot to the other. The resulting walking pattern is illustrated in Fig. 2. The robot moves approximately $4 \mathrm{~cm}$ per step on a laminate floor. With a step frequency of about $2 \mathrm{~Hz}$, this corresponds to a speed of $8 \mathrm{~cm} / \mathrm{s}$. In the second gait mode the step frequency is increased to about $2.7 \mathrm{~Hz}$, but the step length decreases to approximately $2 \mathrm{~cm}$. This results in a speed of about $5.2 \mathrm{~cm} / \mathrm{s}$. RoboSapien walks backwards in a similar way. It can also turn on the spot.

The original RoboSapien is controlled by a human operator who pushes buttons on a remote control. 67 motion commands can be issued to the robot. The motion primitives can be combined, e.g. to have the robot walk a curve. Preprogrammed motion chains can be triggered by touch sensors, located in its feet and at its finger tips, as well as by a sonic sensor which reacts to clapping sounds. 

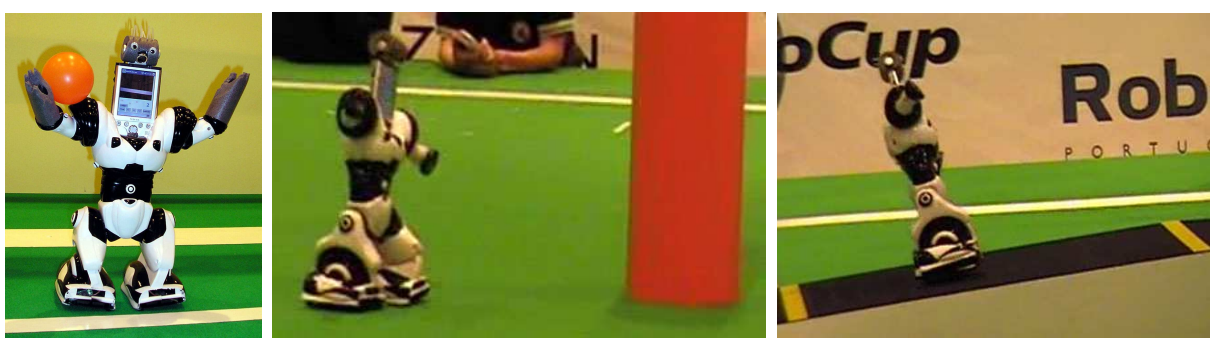

Fig. 3. The augmented RoboSapien competed as NimbRo RS at RoboCup 2004: Humanoid Walk and Balancing Challenge.

\section{Augmented RoboSapien}

In order to make RoboSapien autonomous, we augmented it with computing power and a camera [2]. As NimbRo RS, this augmented RoboSapien took part in some of the RoboCup 2004 Humanoid League competitions (see Fig. 3). It performed the Humanoid Walk and was one of only two robots which mastered the Balancing Challenge, resulting in an overall third place in the Technical Challenges. In order to play soccer, we augmented four more RoboSapiens with an updated Pocket PC and a wide-angle camera as follows (conf. to Fig. 6).

For the Pocket PC, we selected the FSC Pocket Loox 720. It has a weight of only $170 \mathrm{~g}$, including the battery, and features a $520 \mathrm{MHz}$ XScale processor PXA-272, 128MB RAM, 64MB flash memory, a touch-sensitive display with VGA resolution, Bluetooth, wireless LAN, an infrared (IR) interface, and an integrated 1.3 MPixel camera.

In order to place this Pocket PC between the shoulders of the robot, we removed RoboSapien's head (keeping the IR receiver) and cut a rectangular opening into its chest. The Pocket PC can easily be removed to charge the battery and to download programs. Software for it can be conveniently developed on a PC using e.g. Microsoft (Embedded) Visual Studio.

The Pocket PC needs to interface the robot base. We implemented an unidirectional IR interface via a learning remote program (UltraMote) and Windows messages. The Pocket PC can send multiple motion commands per second to the robot base.

Since in RoboCupSoccer key objects, such as the ball and the goals, are colorcoded, visual perception provides a rich source of information about the robot's environment. For this reason, we added a miniature color camera to the Pocket PC. From the few available models, we selected the Lifeview FlyCam-CF 1.3M. An SDK is provided by Lifeview that allows user programs to capture uncompressed live images in the RGB color space. The camera supports resolutions from $160 \times 120$ up to $1280 \times 1024$ pixels. At $320 \times 240$ pixels it delivers 5 fps. We replaced the original camera lens with a ultra-wide angle lens. The field of view of this camera is now about $150^{\circ}$ horizontally $\times 112^{\circ}$ vertically. This allows the augmented RoboSapien to see at the same time its own feet and objects above the horizon (conf. to Fig. 4(a)). 


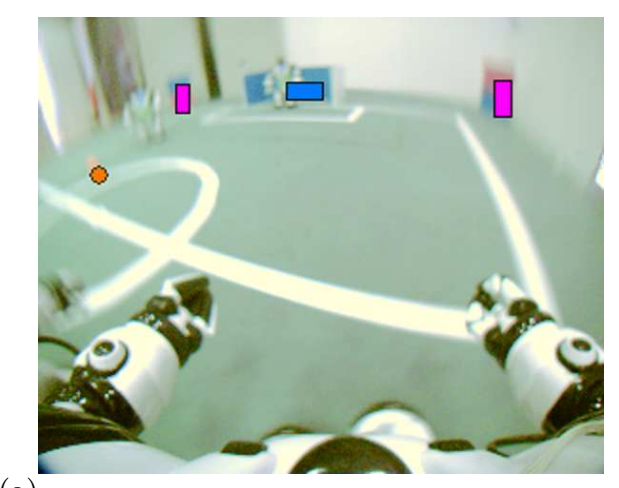

(a)

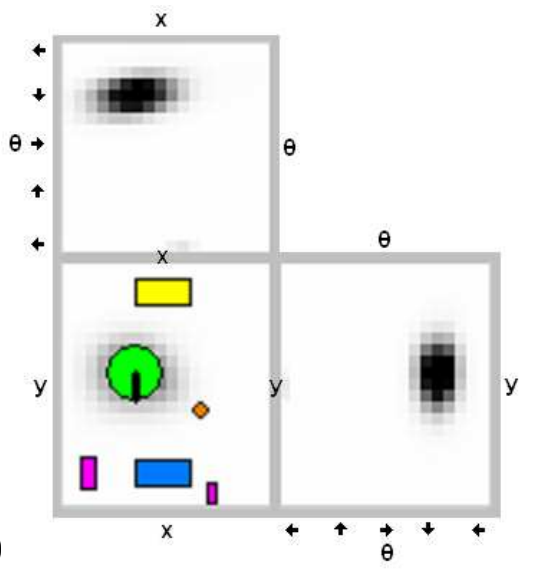

Fig. 4. (a) Image captured from RoboSapien's perspective while it was walking. Detected objects: goal (blue horizontal rectangle), ball (orange circle), and field markers (magenta vertical rectangles); (b) Three two-dimenensional projections of the grid representing the probability distribution of robot poses $(x, y, \theta)$. The green circle is drawn at the estimated robot location $(x, y)$. The black line represents its estimated orientation $\theta$. The detected objects are drawn relative to the robot.

The described modifications make the augmented RoboSapien fully autonomous. The Pocket PC runs computer vision, behavior control, and wireless communication. The total costs for the parts (robot base, Pocket PC, camera, lens, UltraMote) are currently about 700 Euros + tax per robot.

\section{Computer Vision and Self Localization}

The images captured by the CF camera are the only source of information about the state of the world that our robots use. In order to control their behavior, this data must be analyzed.

Our computer vision software converts the captured RGB images into the YUV color space to decrease the influence of different lighting conditions. The colors of pixels are classified with the pie-slice method [22]. In a multistage process insignificant colored pixels are discarded and the colored objects ball, goals, and field markers are detected. Their coordinates are estimated in an egocentric frame (distance to the robot and angle to its orientation). These estimates are based on image positions and object sizes. The robot-centered coordinates suffice for many relative behaviors, like positioning behind the ball while facing the goal, and dribbling the ball.

To implement global team behaviors, such as kick-off, we need the robot coordinates in an allocentric frame (position on the field and orientation). We estimate these using a probabilistic Markov localization method that integrates egocentric observations and motion commands over time. As proposed by Fox, 
Burgard, and Thrun [6] this method uses a three-dimensional grid $(x, y, \theta)$, shown in Figure 4(b).

We use the localization to compute relative coordinates for the goals if they are currently not visible in the image. Robot localization is also needed for the fusion of local robot views to a global team view. We integrate ball observations from multiple robots using a particle filter to obtain a better estimate of the ball position. The fused ball position is used by the robots that do not see the ball themselves. It is also the basis for the assignment of roles to players.

\section{Behavior Control}

Since we cannot change the gaits of RoboSapien, behavior control for it needs to focus on higher-level issues, like ball handling, positioning on the field, and team play.

To simplify the behavior control interface to the robot base, we implemented a set of parameterized motion functions, like walking straight for a distance or turning for a certain angle. The motion functions initiate the movement and wait according to the desired distance or the desired turning angle.

Another possibility is to initiate a movement and to keep moving until a desired state change is reported by our computer vision software. This feedbackcontrol is more robust than feed-forward motion macros, but it relies on the visibility of objects.

We try to move the robots in a way that the key objects (ball, goals, and markers) are in the robot's field of view, but this is not always possible. For example, if the robot wants to move around the ball, the robot has to pass the ball first. The robot can only turn towards the ball again when the ball is lying behind the robot, outside its field-of-view. Another example is the situation where the robot faces the goal and wants to move laterally in order to align itself with the ball and the goal. In this case, the robot has to turn (potentially loosing ball sight), to walk towards the goal-ball line, and to turn back. For such cases, we implemented motion macros that chain up to four motion commands. They are triggered when the ball is leaving the robot's field of view. The robot executes the macros and regains ball sight at the end of a macro.

The rate of behavior decisions is limited by the frame rate of the camera and the rate at which RoboSapien accepts new motion commands. Currently, the entire cycle (image capture, computer vision, self localization, behavior control, and motion command) is executed at about $4 \mathrm{~Hz}$. This is more than sufficient, compared to the speed of the robots and the ball.

Using the described combination of feed-forward and feedback control, we implemented a number of basic soccer skills. If the robots do not see the ball, they wander on the field to search for it. They can approach the ball, such that they face the goal. They can dribble it towards the goal. If the ball is between a robot and our own goal the robot can move around the ball. We also implemented some defensive behaviors for the goal keeper. 
On an external PC, we implemented team behaviors, such as kick-off and normal play. These can assign roles like primary attacker and secondary attacker to the robots. They also can send the robots to an arbitrary position on the field.

\section{Infrastructure}

In addition to the robots themselves, some infrastructure components are needed to support a team of soccer playing robots.

The most obvious of these are the ball and the field. Our robots play with the small orange plastic ball used in the RoboCup Humanoid League. It has a diameter of $8.4 \mathrm{~cm}$ and a weight of $26 \mathrm{~g}$.

The field size of $3.2 \mathrm{~m} \times 2.6 \mathrm{~m}$ also complies to the most recent Humanoid League rules proposal [14]. As playing surface, we use green carpet. The field is marked with $4.8 \mathrm{~cm}$ wide white lines, as sketched in Fig. 5. In addition to the outer field border, we mark a line separating the two field halves, a center circle of $90 \mathrm{~cm}$ diameter, and goal areas of size $120 \mathrm{~cm} \times 40 \mathrm{~cm}$. The goals are $80 \mathrm{~cm}$ wide, $30 \mathrm{~cm}$ deep, and $30 \mathrm{~cm}$ high. They are colored in sky-blue and yellow.

We added markers around the field to aid robot localization. The markers are placed at a $50 \mathrm{~cm}$ distance to the field line. Our first attempt was to use four poles, borrowed from the Aibo-League, placed at the long side of the field, $1 \mathrm{~m}$ from the half line. It turned out that the $10 \mathrm{~cm} \times 10 \mathrm{~cm}$ color patches of these poles were hard to see from a larger distance. For this reason, we switched to six rectangular markers, with color patches of size $29,7 \mathrm{~cm} \times 21 \mathrm{~cm}$ (A4 paper). Two of these patches are placed on top of each other. One is always magenta. The other is white for markers placed in the middle of the long field side. It is yellow or sky-blue for markers placed at the corners. Markers on the left side of the field (when facing the yellow goal) have magenta as upper color. For the right field side, magenta is below the other color.

The Pocket PCs used in the augmented RoboSapiens are equipped with wireless network adapters. We use the wireless UDP communication to transmit debug information to an external computer where it is logged and visualized. This computer is also used to fuse local views to a team view and executes team behaviors.

In order to be able to design behaviors without access to the real hardware, we implemented a physics-based simulation for two teams of RoboSapiens. This simulation is based on the Open Dynamics Engine [18].

\section{Experimental Results}

In addition to the computer vision, self localization, and behavior control components described above, an alternative set of these components has been developed in a lab course by a group of six students.

Both systems are able to play soccer. To evaluate them, we played test games in our lab with an increasing number of players. A similar lab project was done 


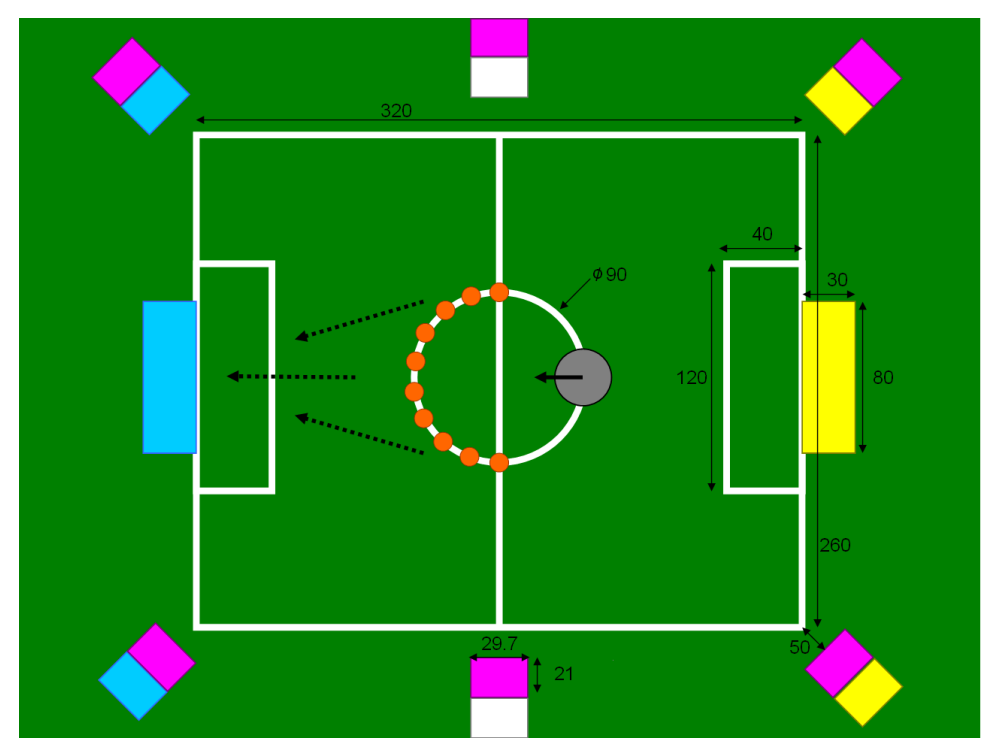

Fig. 5. Scoring Test. Sketch of the field setup, robot and ball positions used.

by the Brainstormers at University of Osnabrück. In April 2005, both teams met at the German Open in Paderborn to show three demonstration games. The games lasted $2 \times 10 \mathrm{~min}$ each and attracted many spectators. During these games, the robots knew most of the time where the ball was. The scored goals were not accidental, but the results of intentional actions. The robots were playing without human help. Only the referee was allowed to touch the robots in order to untangle them in the case of entanglements. Whenever the ball went outside the field, the referee would put it back to the field line at the position where it left the field. We also learned that the presence of more than two robots in the goal box (one attacker and the goalie) must be prevented by the rules to avoid overcrowding.

In order to produce a performance estimate that is less noisy and easier to obtain than the score of entire soccer games, we designed a scoring test, illustrated in Fig. 5. In this test, one robot stands on the most distant point of the center circle, facing the empty goal, which is $2.05 \mathrm{~m}$ away. The ball is placed at ten different positions on the half of the center circle which is closer to the goal $\left(20^{\circ}\right.$ steps $)$. The chosen ball position is not communicated to the robot. Its task is now to bring the ball into the goal as quickly as possible.

Our system managed to score in all ten trials. The augmented RoboSapien needed on average 170 s to score. The robot was operating continuously during the scoring test. The only human intervention was to place the ball and the robot at their initial positions.

For comparison, a human-controlled (via the original remote control unit) augmented RoboSapien (perfect perception, almost perfect behavior control) took on average $97 \mathrm{~s}$ for scoring. It had a $100 \%$ success rate as well. 

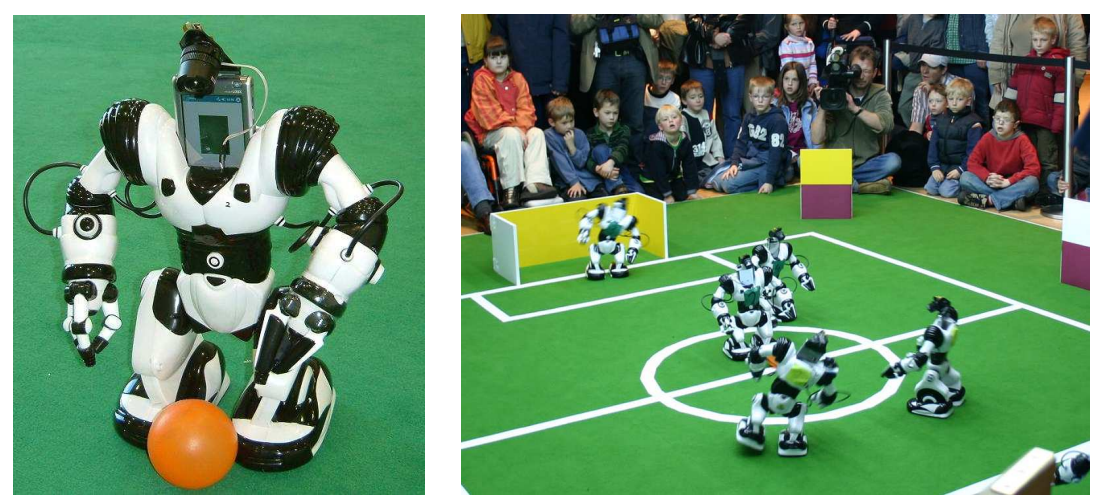

Fig. 6. Augmented RoboSapiens playing soccer at RoboCup German Open 2005.

\section{Conclusions}

In this paper, we described a way to augment low-cost commercial off-the-shelf humanoid robots in order to convert them into a soccer team.

For programmable autonomy, we attached Pocket PCs to the RoboSapiens. They provide ample computing power and have many interfaces. To allow for visual perception of the game situation, we added a color CMOS camera.

We implemented computer vision, probabilistic self-localization, and behavior control on the Pocket PC. In addition, we set up a soccer field, wireless communication, and a physics-based simulation.

This system was able to play test games in our lab and at German Open 2005. The augmented RoboSapien also performed well in a scoring test. The soccer experiments revealed some limitations of the augmented RoboSapien. They include low precision, unidirectional IR communication, and mechanical limitations. The low precision of walking makes it unfeasible to rely on path integration for navigation. It is necessary to compensate for the quickly accumulating deviations by visual feedback. The unidirectional IR communication from the Pocket PC to the robot base prevents the use of proprioceptive information, touch sensors, and sonic sensors for behavior control. The low number of DOFs as well as the low center of mass limit the possible movements. For instance, while it is possible to dribble a ball with the robot, RoboSapien is unable to perform the powerful kick needed for penalties. It is also unable to walk laterally, but must turn towards the target before walking.

Despite these limitations, we think that augmented RoboSapiens are a suitable platform for performing multi-robot experiments with humanoid robots. When working with such a platform, one does not need to deal with the difficulties of bipedal walking and balance. Since these issues are solved by the RoboSapien base, the researchers can focus on visual perception and higher-level behavior control.

As can be seen in the RoboCup Four-legged (Aibo) League, the use of standardized hardware has certain advantages and disadvantages. On the positive 
side, there is no need to develop and build robots for researchers interested in perception and behavior control. One can start with an off-the-shelf robot to develop software. Standardized hardware also facilitates the exchange of software components and the comparison of experimental results between research groups.

On the other hand, commercial robots are usually not fully open. The developer has to use the API provided by the manufacturer and cannot modify the software and hardware layers below the API. These changes are done exclusively by the manufacturer, which might limit the exploration of new ideas by researchers. While it is in many cases possible to find a work around a limitation, this approach might lead to the use of the hardware in a way not intended by the manufacturer. One example for this is the walking on the knees (instead of the paws) adopted by most of the participants of the RoboCup Four-legged League.

For the reasons above, we think that the availability of capable standard hardware would facilitate empirical multi-agent research on humanoid robots. If such robots were open and well documented, they could be used as a starting point for researchers. One step towards this goal was to augment RoboSapien.

Since the costs for this programmable autonomous humanoid robot are only about EUR 700, it is feasible to perform experiments with more than one robot even for research groups lacking huge resources. This could be interesting not only for university groups and industry labs, but also for RoboCup Junior, education, and enthusiasts.

We made the API for sending motion commands to the robot base and capturing images, as well as a tutorial how to augment RoboSapien publicly available. Other research groups adopted the augmented RoboSapien already. We delivered one robot to Microsoft Research Cambridge. The Brainstormers of University of Osnabrück also augmented a number of RoboSapiens. Together, we played three soccer demonstration games at the RoboCup German Open (April 2005) in Paderborn. At RoboCup 2005, the team Hiro used augmented RoboSapiens in the Humanoid League competitions.

Videos of RoboSapiens playing soccer and more images can be found on our website: http://www.NimbRo.net [/rs].

\section{Acknowledgements}

This project is funded by grant BE 2556/2-1 of Deutsche Forschungsgemeinschaft (German Research Foundation, DFG). We would like to thank Mark W. Tilden and WowWee for providing two RoboSapien samples.

Jörg Stückler helped us with the simulator. Tobias Langner worked on the RoboSapien API. Dr. Maren Bennewitz provided advice on probabilistic localization. Andreas Maunz, Kai M. Wurm, Jens Singler, Markus Ihmsen, Marc Gißler, and Jan Kurrus developed the alternative system in a lab project.

\section{References}

1. Fraunhofer AIS. VolksBot. http://www.ais.fraunhofer.de/be/volksbot/. 
2. Sven Behnke, Tobias Langner, Jürgen Müller, Holger Neub, and Michael Schreiber. NimbRo RS: A low-cost autonomous humanoid robot for multi-agent research. In Proc. of WS on Methods and Technology for Empirical Evaluation of Multi-Agent Systems and Multi-robot Teams (MTEE) at KI2004, Ulm, Germany, 2004.

3. Sylvain Calinon and Aude Billard. PDA interface for humanoid robots. In Third IEEE International Conference on Humanoid Robots (Humanoids 2003), 2003.

4. CMU. Palm Pilot Robot Kit. http://www-2.cs.cmu.edu/ reshko/pilot/.

5. Stefan Enderle, Stefan Sablatnög, Steffen Simon, and Gerhard K. Kraetzschmar. Tetrixx - A Robot Development Kit. In Proc. of Edutainment Robots WS, 2000.

6. Dieter Fox, Wolfram Burgard, and Sebastian Thrun. Markov localization for mobile robots in dynamic environments. Journal of Artificial Intelligence Research, 11:391-427, 1999.

7. Fujitsu. HOAP-2. http://www.automation.fujitsu.com/en/products/products09.html.

8. Honda. ASIMO. http://world.honda.com/asimo/.

9. Thomas Hsiu, Steve Richards, Ajinkya Bhave, Andres Perez-Bergquist, and Illah Nourbakhsh. Designing a low-cost, expressive educational robot. In Proc. of Int. Conf. on Intelligent Robots and Systems (IROS), Las Vegas, 2003.

10. iXs Research Corp. http://www.ixs.co.jp.

11. Alexander Koller and Geert-Jan Kruijff. Talking robots with LEGO mindstorms. In Proc. of 20th Int. Conf. on Computational Linguistics (COLING), Geneva, 2004.

12. Kondo Kagaku Co., Ltd. KHR-1. http://www.kondo-robot.com.

13. Henrik Hautop Lund and Luigi Pagliarini. RoboCup Jr. with LEGO Mindstorms. In Proc. of Int. Conf. on Robotics and Automation, San Francisco, CA, 2000.

14. Norbert M. Mayer. Humanoid Kid Size League and Medium Size League rules and setup. http://er04.ams.eng.osaka-u.ac.jp/humanoid_webpage/humanoid.pdf.

15. Kevin Mukhar, Dave Johnson, Kevin Mukhar, and Dave Johnson. The Ultimate Palm Robot. McGraw-Hill, 2003.

16. Masaki Ogino, Masaaki Kikuchi, Junichiro Ooga, Masahiro Aono, and Minoru Asada. Optic flow based skill learning for a humanoid to trap, approach to, and pass a ball. In RoboCup 2004: Robot Soccer World Cup VIII, pages 323-334, 2005.

17. Greg Reshko, Matthew Mason, and Illah Nourbakhsh. Rapid prototyping of small robots. Technical Report CMU-RI-TR-02-11, Robotics Institute, Carnegie Mellon University, Pittsburgh, PA, March 2002.

18. Russel Smith. Open Dynamics Engine. http://opende.sourceforge.net.

19. Sony. Dream Robot QRIO. http://www.sony.net/qrio.

20. Speecys Corp. Speecys robot kit. http://www.speecys.com.

21. Team Osaka. VisiON. http://www.sansokan.jp/robot/info/vision_en.html.

22. Peter J. Thomas, Russel J. Stonier, and Peter J. Wolfs. Robustness of colour detection for robot soccer. In Proc. of 7th Int. Conf. on Control, Automation, Robotics and Vision (ICARCV), volume 3, pages 1245-1249, 2002.

23. Mark W. Tilden. Neuromorphic robot humanoid to step into the market. The Neuromorphic Engineer, 1(1):12, 2004.

24. Toyota. Partner Robot. http://www.toyota.co.jp/en/special/robot/.

25. Tribotix Pty Ltd. Cycloid. http://www.tribotix.com/products/robotis/cycloid.htm.

26. Vstone Co., Ltd. http://www.vstone.co.jp.

27. Doug Williams. PDA Robotics. McGraw-Hill, 2003.

28. Chris Willis. World's greatest android projects. http://www.androidworld.com.

29. Xudong Yu and Jerry B. Weinberg. Robotics in education: New platforms and environments. IEEE Robotics \& A Atomation Magazine, 10(3), 2003.

30. Changjiu Zhou and Pik Kong Yue. Robo-Erectus: a low-cost autonomous humanoid soccer robot. Advanced Robotics, 18(7):717-720, 2004. 